\title{
Piloplex, a new long-acting pilocarpine polymer salt. B: Comparative study of the visual effects of pilocarpine and Piloplex eye drops
}

\author{
ZEEV MAZOR, ${ }^{1}$ URIEL TICHO, ${ }^{2}$ URI REHANY, ${ }^{2}$ AND LIONEL ROSE ${ }^{2}$ \\ From the ${ }^{1}$ Hydrophilics Laboratories, Haifa, and the ${ }^{2}$ Department of Ophthalmology, Hadassah Hospital, \\ Jerusalem, Israel
}

SUMMARY Induced accommodation and changes in vision (distance and near) were measured monocularly and binocularly in 9 young healthy volunteers in a double blind study after administering to them pilocarpine hydrochloride $4 \%$, Piloplex $3 \cdot 4$, and saline eye drop instillations. Piloplex $3 \cdot 4$, a new long-acting pilocarpine polymer salt, and pilocarpine hydrochloride $4 \%$ (both contain equal amounts of pilocarpine-3.4\%) induced changes in vision and accommodation. These changes were greater with pilocarpine hydrochloride than with Piloplex. The maximum changes occurred half an hour after instillation and the effect vanished after an additional period up to 3 hours. The changes were greater when measured monocularly than binocularly. Piloplex initiates a prolonged hypotensive effect which lasts for 12 hours. Patients with glaucoma are thus able to use Piloplex on a twice-daily schedule. Consequently, visual disturbances occur only once a day in contrast to pilocarpine hydrochloride given 4 times a day, which induces 3 visual disturbances during the day.

The advantage of Piloplex - a new pilocarpine polymer salt - over pilocarpine hydrochloride $(\mathrm{PHCl})$ has been previously presented (Ticho et al., 1978; Blumenthal et al., 1979). Piloplex eye drops were found to have a prolonged and increased hypotensive effect, allowing them to be given on a twice-daily schedule in contrast to a 4-times daily schedule necessary for $\mathrm{PHCl}$. The hypotensive effect of miotic drugs, however, is accompanied by miosis and accommodative spasm, inducing blurred vision. These side effects are predominant in young patients (Place et al., 1975; Brown et al., 1976). Adverse side effects of this nature have not been encountered with Piloplex treatment so far.

This communication reports on a study in which the induced visual effects of pilocarpine hydrochloride and Piloplex were compared in young normal persons.

\section{Patients and methods}

Nine medical students who had no record of ophthalmic disturbances were selected for the study.

Address for reprints: Dr U. Ticho, Department of Ophthalmology, Hadassah Hospital, Jerusalem, Israel
On ophthalmic examination before the study all the students had 20/20 visual acuity in both eyes, 4 of them having spectacle corrections. Their informed consent was obtained after a detailed explanation of the study.

The group consisted of 7 men and 2 women, ranging in age from 24 to 31 years. Three of them had green eyes, 3 had brown eyes, 2 had grey-blue eyes, and 1 had hazel eyes. They were examined during 3 daily sessions and were assigned randomly to 3 different eye drop treatments: (1) Pilocarpine hydrochloride 4\%; (2) Piloplex 3.4 (Hydrophilics); and (3) isotonic saline. The first 2 eye drop preparations contained equivalent amounts of pilocarpine ion. In each session 1 eye drop was instilled into each eye (both eyes were treated similarly). Successive sessions with different medications were performed 3 or 4 days apart.

All examination sessions included subjects receiving all 3 types of treatments. Subjects were examined in random order. Neither the tested subject nor the examiner knew which of the medications were tested at each examination.

The examinations were of visual acuity and induced accommodation. The visual acuity was determined at $20 \mathrm{ft}(6 \mathrm{~m})$ by a Snellen projector 
Fig. 1 Average changes in visual acuity of 9 normal persons after pilocarpine hydrochloride $4 \%$, Piloplex 3.4, and saline eye drops
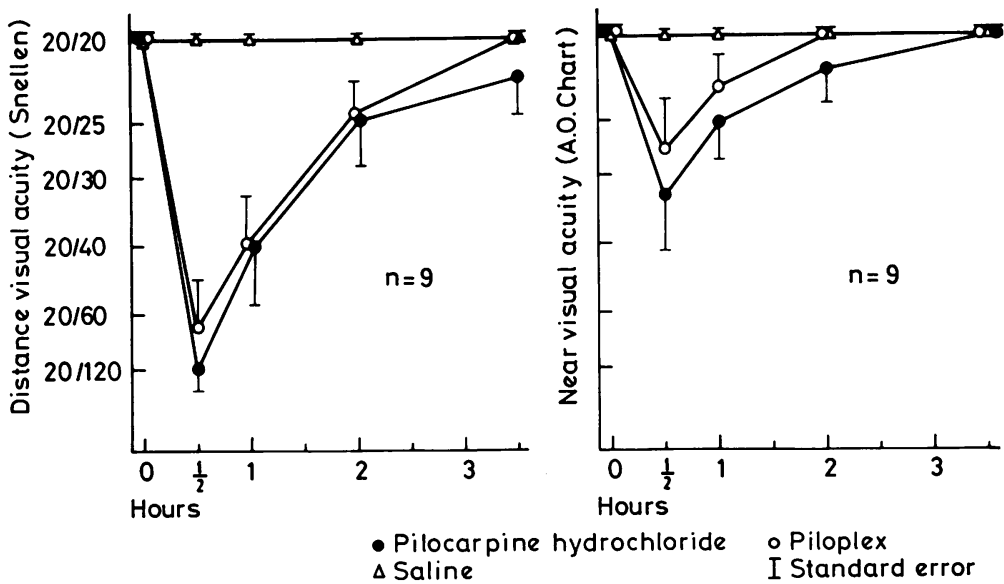

chart of numbers. Near vision was evaluated at 13 in $(33 \mathrm{~cm})$ with the American Optical Company nearpoint chart (AO). The induced accommodation was estimated by measuring the 'plus to blur' error. Where there was a reduction of visual acuity, normal vision was restored by adding minus lenses. The amount of minus power was then reduced until the person again reported blurred vision. The difference in the power of the lenses to induce blurring before and after medication was considered to be the amount of induced accommodation. Measurements of visual acuity and induced accommodation were performed for distance and near vision with each eye separately and with both eyes together.

All examinations were performed immediately before medication (zero time) and at 30, 60, 120, and 210 minutes after the administration of eye drops. Results were statistically examined by the Wilcoxon matched pairs signed rank test.

\section{Results}

DISTANCE AND NEAR VISION

Vision was reduced in the 9 students at distance and near vision after instillation of both types of pilocarpine eye drops (Fig. 1). The maximum reduction was half an hour after instillation. Distance vision returned to normal after a further 3 hours, while near vision returned to normal 2 hours after instillation of Piloplex and $3 \frac{1}{2}$ hours after instillation of $\mathrm{PHCl}$.

PHCl induced a larger visual effect than Piloplex, but the difference was not significant. The visual effect caused by both pilocarpine medications was stronger when measured on a single eye than on both eyes. Distance vision was reduced to less than
20/100 30 minutes after instillation of $\mathrm{PHCl}$ in 6 out of the 9 persons when measured with both eyes open. However, when the vision of each eye was measured separately it was reduced to $20 / 100$ in 16 out of 18 eyes 30 minutes after instillation. Near vision was reduced to less than 20/50 30 minutes after instillation of $\mathrm{PHCl}$ in 3 out of the 9 persons, while 9 out of the 18 eyes showed similar reduction of vision at the same time.

INDUCED ACCOMMODATION MEASURED AT DISTANCE AND NEAR

Induced accommodation was measured in all 9 students at distance and near vision after instillation of both types of pilocarpine eye drops. The maximum change in induced accommodation was reached 30 minutes after instillation and gradually wore off during the next 3 hours (Fig. 2). $\mathrm{PHCl}$ caused a larger change in accommodation than Piloplex, but the difference was not significant. Change in accommodation was greater when measured monocularly than with both eyes open at distance (Fig. 3) and at near vision with both medications.

Changes in vision and induced accommodation were fairly consistent throughout the study with both medications. Persons who showed the greatest change on induced accommodation also showed the greatest changes in distance and near vision.

\section{Discussion}

Pilocarpine causes hypotensive and visual effects which can be measured by changes in intraocular pressure, vision (distance and near), refraction, and miosis. Changes in vision and refraction, however, are not directly dose-related to the concentration of pilocarpine in the eye. Thus, constant delivery of 


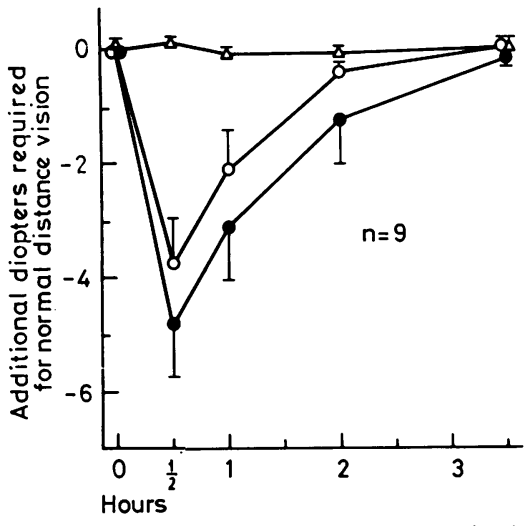

- Pilocarpine hydrochloride $\Delta$ Saline

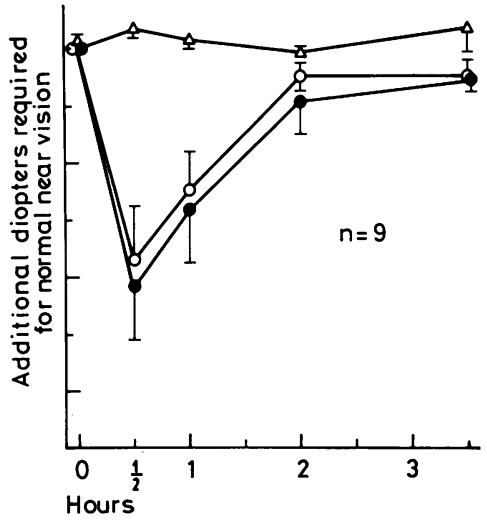

- Piloplex

I Standard error
Fig. 2 Average amount of induced accommodation. expressed as 'plus to blur' measurement for distance vision (left) and near vision (right) of 9 normal persons after pilocarpine hydrochloride $4 \%$, Piloplex $3 \cdot 4$, and saline eye drops

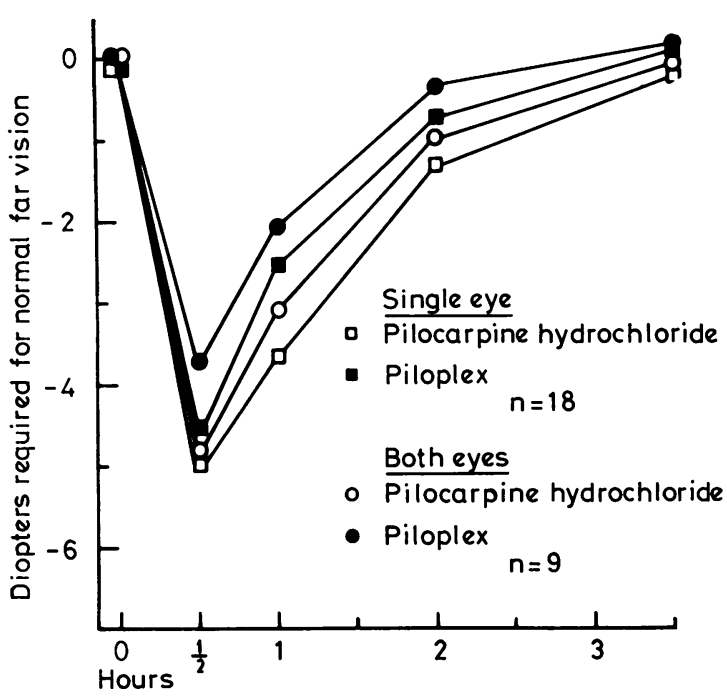

Fig. 3 Average induced accommodation measured monocularly and with binocular vision of 9 normal persons after pilocarpine hydrochloride $4 \%$ and Piloplex 3.4 eye drops

small amounts of pilocarpine $(20 \mu \mathrm{g} / \mathrm{h}$ by Ocusert) produced visual effects-refraction, distance and near vision-which did not substantially differ from those initiated by delivery of $40 \mu \mathrm{g} / \mathrm{h}$ of pilocarpine. Miosis and hypotensive effects were stronger after higher doses (Brown et al., 1976). Thus miosis and ocular hypotension appear to be more sensitive to relatively low doses of pilocarpine than refractive changes and distance and near vision.

Changes in all parameters are reported when the amount of pilocarpine is increased, ranging from 1 to $4 \%$, and the changes were dose-related (Brown et al., 1976). It is expected that saturation of the pilocarpine binding sites will create the maximum visual effect, and higher doses will cause no further changes. Quigley and Pollack (1977) reported experiments with 2 different viscous preparations of $\mathrm{PHCl}$ (same pilocarpine dose). Green and Downs (1975) when using these preparations found different pilocarpine levels in rabbit aqueous humour. However, no significant intraocular pressure difference was reported comparing the hypotensive effect of these 2 different preparations in patients (Quigley and Pollack, 1977).

The present study shows that very similar changes in refraction and far and near vision were induced by Piloplex and $\mathrm{PHCl}$, thus hinting that during the first period after instillation of the drug the amount of pilocarpine in the eye is much the same from each of the two pilocarpine preparations. However, the fact that Piloplex causes a longer hypotensive effect than $\mathrm{PHCl}$ (Ticho et al., 1978; Blumenthal et al., 1979) suggests that later on after instillation the amount of pilocarpine in the eye from $\mathrm{PHCl}$ is no longer sufficient to maintain a hypotensive effect. In contrast, in the later period after instillation of Piloplex there is sufficient pilocarpine in the eye to provide a longer-lasting hypotenstive effect but without inducing visual disturbances. A similar situation was reported for Ocusert (Brown et al., 1976).

Piloplex, a salt, releases pilocarpine ion at a sustained rate (Ticho et al., 1978) and initiates a prolonged hypotensive effect lasting 12 hours; it therefore can be given on a twice daily schedule. Since adverse visual effects after evening medication (instilled before night sleep) do not disturb the patient, it could be concluded that Piloplex induces visual disturbances only once a day (after the 
morning medication) in contrast to $\mathrm{PHCl}$ on a 4 times daily schedule, which induces 3 disturbances during the daytime.

We thank Mario Baras, of the Department of Medical Ecology of the Hebrew University, Hadassah Medical School, Jerusalem, for his statistical aid.

\section{References}

Blumenthal, M., Ticho, U., Zonis, S., Gal, A., Blank, I., and Mazor, Z. W. (1979). Further clinical trial with Piloplexa new long acting pilocarpine salt. Annals of Ophthalmology (in press).

Brown, H. S., Meltzer, G., Merrill, R. C., Fisher, M., Ferré,
C., and Place, V. A. (1976). Visual effects of pilocarpine in glaucoma. Archives of Ophthalmology, 94, 1716-1719.

Green, K., and Downs, S. T. (1975). Ocular penetration of pilocarpine in rabbit. Archives of Ophthalmology, 93, 1165-1168.

Place, V. A., Fisher, M., Herbst, S., Gordon, L., and Merrill, R. C. (1975). Comparative pharmacologic effects of pilocarpine administered to normal subjects by eye drops or by ocular therapeutic systems. American Journal of Ophthalmology, 80, 706-712.

Quigley, H. A., and Pollack, I. P. (1977). Intraocular pressure control with twice daily pilocarpine in two vehicle solutions. Annals of Ophthalmology, 9, 427-429.

Ticho, U., Blumenthal, M., Zonis, S., Gal, A., Blank, I., and Mazor, Z. W. (1978). A new long-acting pilocarpine compound. Annals of Ophthalmology (in press). 